\title{
Plan integral de mejora para fortalecer la gestión hospitalaria a nivel institucional en la región San Martin, 2021
}

\author{
Jaime Flores Arévalo \\ diego29_4@hotmail.com \\ https://orcid.org/0000-0002-5165-5897 \\ Escuela de Posgrado Universidad César Vallejo \\ Hipólito Percy Barbarán Mozo \\ pbarmozito@hotmail.com \\ https://Orcid.org/0000-0002-9316-202X \\ Escuela de Posgrado Universidad César Vallejo
}

\section{RESUMEN}

La investigación presenta por objetivo sistematizar el plan integral de mejora para fortalecer la gestión hospitalaria a nivel institucional en la región San Martín. La investigación es de tipo básica y proyectiva, con diseño no experimental a nivel descriptivo, teniendo como muestra a 110 colaboradores profesionales y técnicos del Hospital EsSalud Tarapoto; a quienes se aplicó un cuestionario mediante la encuesta para la fase diagnóstica; a su vez, se aplicó un test para recabar opinión de los expertos sobre la propuesta. Los principales resultados muestran que, algunos aspectos de la gestión hospitalaria han sido considerados como regular predominantemente según los encuestados; gestión clínica (72\%), gestión administrativa (63\%), gestión de recursos humanos (85\%), gestión de recursos tecnológicos (66\%), gestión del sistema de información (69\%), investigación y docencia (57\%), financiamiento y mecanismo de pago (81\%), etc.; y la propuesta fue calificada como adecuada y excelente. En conclusión, la sistematicidad del plan integral de mejora para fortalecer la gestión hospitalaria a nivel institucional en la región San Martín, 2021, es muy adecuado (46\%) y posee excelente contribución (70\%) para resolver la problemática y prospectiva de realizarse $(60 \%)$, según la opinión de los expertos; es decir, es viable, factible, aplicable, sostenible y pertinente.

Palabras clave: plan integral; gestión hospitalaria; sistema de salud. 


\title{
Integral improvement plan to strengthen hospital management at the institutional level in the San Martin region, 2021
}

\begin{abstract}
The objective of the research is to systematize the comprehensive improvement plan to strengthen hospital management at the institutional level in the San Martín region. The research is of a basic and projective type, with a non-experimental design at a descriptive level, having as a sample 110 professional and technical collaborators of the Hospital EsSalud Tarapoto; to whom a questionnaire was applied through the survey for the diagnostic phase; in turn, a test was applied to obtain the opinion of the experts on the proposal. The main results show that some aspects of hospital management have been considered as predominantly regular according to the respondents; clinical management (72\%), administrative management (63\%), human resource management (85\%), technological resource management (66\%), information system management (69\%), research and teaching (57\%), financing and payment mechanism (81\%), etc.; and the proposal was rated as adequate and excellent. In conclusion, the systematic nature of the comprehensive improvement plan to strengthen hospital management at the institutional level in the San Martín region, 2021, is very adequate (46\%) and has an excellent contribution (70\%) to solve the problems and prospects to be carried out (60\%), according to the opinion of the experts; that is, it is feasible, feasible, applicable, sustainable and relevant.
\end{abstract}

Keywords: comprehensive plan; hospital management; health system.

Artículo recibido: 20 diciembre. 2021 Aceptado para publicación: 10 enero 2022 Correspondencia: diego29_4@ hotmail.com Conflictos de Interés: Ninguna que declarar 


\section{INTRODUCCIÓN}

Gestión Hospitalaria [GH] conceptualmente se define como aquella función que es básica en la sucesión administrativa hospitalaria, permitiendo la optimización de la oferta de servicios hospitalarios ante una necesidad de demanda de atención sanitaria, en donde la planeación correcta sumada a la gestión objetiva posibilitan la toma de decisiones y la gestión de la administración sea oportuna y eficiente (López y López, 2019). Bajo este concepto, la salud representa un derecho constitucional, donde la atención sanitaria es articulada entre los sectores públicos y privados tal que permite atender las diferentes clases sociales, debido a que, la calidad de los servicios de la salud es vista con criterios orientados al interés del usuario.

Henríquez (2010), en su investigación, detalla que el sistema de salud público en Chile constantemente ha estado expuesto a cuestionamientos; por la existencia de situaciones que juegan en contra de indicadores hospitalarios, como: el manejo de las listas de espera, el déficit de equipamiento e insumos médicos en los hospitales, la falta de especialistas, el colapso de las urgencias, el estancamiento en la producción (que ha derivado, por ejemplo, en el aumento de las contrataciones intrahospitalarias de prestadores privados y sociedades médicas para cubrir el exceso de demanda), entre otros, que constituyen elementos de gestión que son objeto de revisiones constantes. El sistema se encuentra especialmente sensible, considerando que dada la contingencia se espera una presión importante en la red asistencial, y que deberá ser abordada por el Presupuesto 2021. De hecho, las atenciones no realizadas en el sistema público, por efecto de la actividad hospitalaria concentrada en la demanda en las atenciones de Covid19, producirán una presión sumamente compleja al alza del gasto en salud.

Saturno et al. (2017), en su investigación, detalla que los sistemas de salud (SS) surten ante una organización de respuesta social con fines de la atención de las prioridades sanitarias de la población así como la promoción del desarrollo en su plenitud, evolucionando hacia la adaptación de los cambios epidemiológicos, económicos, políticos, sociales y demográficos; en efecto, la esencia fundamental de esta evolución es la calidad de la prestación. En consecuencia la creciente manifestación saludable en los mexicanos es por los logros obtenidos de los sistemas de salud.; no obstante, al analizar indicadores de desempeño del sistema sanitario y realizando comparación con otras naciones, México con respecto a la mayoría de naciones que forma parte de la 
Organización para la Cooperación y el Desarrollo Económico (OCDE) se encuentra ubicado muy por debajo e, inclusive ante unos cuantos de América Latina; explicando que el sistema sanitario confronta barreras importantes en la prestación de salud con calidad. Es un hecho que la calidad difiere importantemente entre grupos poblacionales, sectores y regiones geográficas. De manera inadecuada las acciones sanitarias, a menudo no son ejecutadas poniendo en un riesgoso juego la salud de los usuarios.

La situación de acceso a la salud en el Perú no es uniforme y equitativa; no uniforme por la existencia de sistemas de salud distintos como Ministerio de Salud, Seguridad Social del Perú, Policía Nacional del Perú cuyas estructuras administrativas y gerenciales no comulgan ante una demanda pertinente de atención de los usuarios, y a su vez inherente como un derecho humano la cual debe ser protegida por el estado y la carta magna; no equitativa porque no todos los peruanos tienen acceso a los servicios por limitación económica y acceso geográfico, además de existir establecimientos con diferentes niveles resolutivos y que muchos no cuentan el equipamiento médico, insumos y medicamentos, recursos humanos asistenciales necesarios; ante todo, la comunidad como acreedor de su bienestar es merecedor que el modelo de gestión hospitalaria existente encamine la unificación administrativa y gerencial de la salud pública, que al parecer no se ejecuta según lo planificado en sus procesos de la macrogestión, mesogestión y microgestión (Flores, 2021)

La atención de la salud a nivel privado tiene otra connotación de su manejo donde los usuarios que demandan son aquellos con economía más pudiente; no obstante, esta forma de administración no exime que el estado pueda intervenir en su control como exigencia de calidad mediante la Superintendencia Nacional de Salud - SUSALUD. Por lo visto, al existir diferentes establecimientos de salud con aparato y normas administrativas, marco jurídico, derechohabientes, e incluso protocolos de atención distintos coadyuvan que la gestión sanitaria u hospitalaria tengan deficiencia en el desarrollo de sus procesos, en efecto no contribuye a una atención integral y universal de la salud. Por tanto, contar con normas y directrices diferentes hace que el desarrollo organizacional en particular mengüe la calidad y la satisfacción de oferta no solo externo, también interno (Flores, 2021)

El Ministerio de Salud - MINSA al crear un modelo de gestión hospitalaria, norma un documento técnico el 2007 tras un estudio de la realidad de los establecimientos públicos 
en gestión hospitalaria, producto de ello reportó como resultado de la evaluación tres componentes procesos, estructura, y resultados todos sobre un estándar del $90 \%$ en su evaluación; resultando el de procesos con el perfil más bajo con 56.8\% de desarrollo de los tres componentes respecto a la gestión hospitalaria. En la evaluación final, sólo el 6 \% alcanzó el nivel de bueno y la mayor parte el 70 \% se gradúa de regular y el $25 \%$ requiere de intervención; es decir, mayor trabajo. Esta evaluación pone en manifiesto que existe una gestión hospitalaria pública nada productiva para el estado y poco beneficiosa para la población demandante (MINSA, 2009).

Si bien existen modelos de gestión hospitalaria ya establecidas, aún existen deficiencia en los resultados de los indicadores obtenidos sobre el desempeño y la calidad en la gestión hospitalaria; y la observada en la evaluación del 2005 del modelo de gestión hospitalaria con los resultados reportados el 2007 por el MINSA donde el componente proceso alcanzó un menor desarrollo de sus diferentes indicadores de gestión; por su parte, Ríos (2016), en su estudio reportó que el componente resultado fue el menos desarrollado, seguido por el de procesos; por cuanto, se observa que aún existen diferencias en el desarrollo de la gestión hospitalaria en el país. Por lo que, la gestión hospitalaria a nivel institucional, demanda la mejora en el fortalecimiento de los procesos en todo sus componentes desarrollando acciones de planes o proyectos en su integralidad de todo el sistema; y para efectos es preciso tener un diagnóstico actualizado y real del entorno externo e interno y plantearse su planificación de mejora. Bastani et al. (2021), en su investigación, donde intervinieron 12 profesionales de un hospital con formación en gestión de servicios de salud, realizado; concluyeron que, el cambio del uso de gerentes tradicionales al de egresados de Gestión de Servicios de Salud en el hospital, propuesto como paradigma de cambio en la gestión hospitalaria, se acompaña de algunas reformas en las estructuras, procesos, recursos del hospital, cultura y desempeño; y manifiesta que tales reformas pueden conducir a valiosos resultados finales, como el aumento de la satisfacción del paciente y del personal y la eficacia de los servicios.

La revolución en la gestión hospitalaria requiere nuevos procedimientos y un cambio de paradigma en el mundo altamente competitivo de hoy. Los hospitales tendrán más éxito si documentan cualquier procedimiento sistemático y bien organizado. Esta preocupación es fundamental para los directores de hospitales de todo el mundo sin importar la nacionalidad. Se considera la mejora del sistema de gestión hospitalaria haciendo más 
hincapié en el sistema hospitalario y, en consecuencia, en la gestión hospitalaria exitosa y las estrategias competitivas. La estrategia competitiva en la gestión hospitalaria ha cambiado de diferentes maneras al enfocarse más en sus habilidades gerenciales y la necesidad de gerentes de proyectos profesionales (Ghasemi et al., 2017).

Saeed (2020), en su investigación encontró que, la gestión hospitalaria realiza y lleva a cabo multitud de operaciones y actividades en diferentes niveles, por tanto, la alta dirección del hospital debe esforzarse por comenzar a implementar una garantía de calidad o implementar una norma ISO 9001: 2008 sistema de gestión de la calidad para alcanzar el tercer nivel de madurez, que es el nivel deseado.

Gu et al. (2019), en su investigación; concluyeron que, la implementación de un sistema de gestión de conocimientos basado en casos (CBHKS) influye en el desempeño del grupo, la satisfacción de los miembros del grupo, el aprendizaje del grupo y la satisfacción externa; y la satisfacción de los miembros del grupo y la satisfacción externa afectan de manera significativa y positiva el desempeño y el mantenimiento de la administración; por lo que se deduce que una gestión en el caso de la hospitalaria, la administración que es uno de sus componentes se afecta su desempeño cuando no hay satisfacción interna (colaboradores) y satisfacción externa (usuarios); en efecto, es importante que cualquier proceso de gestión que se implemente se tome en cuenta tanto al cliente interno y cliente externo; de éste dependerá la calidad de los resultados en cuanto a gestión hospitalaria, inclusivamente.

Mei y Kirkpatrick (2019), en su investigación; concluyeron que, son poco los autores que en la reforma de la nueva gestión pública (NGP) consideren la atención médica, por lo que las reformas actuales deben revertir parcialmente; no extender, la tendencia hacia la mercantilización para fortalecer la orientación pública de los hospitales públicos. En segundo lugar, hay una brecha marcada entre la retórica y la realidad de empoderar a los gerentes y liberarlos del control político. Esta contribución investigativa refleja más la existencia de la mercantilización de la salud y no la prestación propia de la salud como derecho humano; además se deduce que en la nueva gestión pública o también conocido como macrogestión o gestión pública hospitalaria, MINSA (2009); existe un control o injerencia política que no permite desarrollar la gestión de los gerentes por existir normas o marcos legales limitantes o restrictivos y con sanciones penales en caso de no acatar; 
muchas veces necesidades o prioridades de atención o acciones que impiden cumplir afectando logros o metas.

Cabe resaltar que en la mejora de los sistemas de sistemas de gestión y en los hospitales la innovación tecnológica y sistemas de información son de mucha relevancia; Naranjo (2016), en su estudio concluyó que, el uso interactivo de los sistemas de información y control de gestión es un intermediario esencial entre la diversificación del grupo direccional y el incentivo de políticas de colaboración y concertación de los establecimientos hospitalarios; además, los grupos con característicamente diversificado como educación, experiencia, antigüedad y edad, etc., tienen la tendencia de hacer uso los sistemas de información de la gestión interactivamente para la mejora de la concertación de actividades y la consignación de los recursos hospitalarios; y Flores (2021) en su estudio; concluyó que, los procesos de mejora en la gestión de los hospitales garantizan que la estandarización resulte en la optimización de la atención, producto de la transformación de los insumos en output como en sí es el bienestar de las personas; entiéndase estandarización como tecnología.

En la perspectiva del paciente la calidad de los servicios de salud puede ser interpretada con diferentes visiones, como decir que calidad de atención es ser atendido en tiempo breve y preciso, y otros decir que calidad de servicio es satisfactoria cuando el profesional invierte su tiempo en escuchar al paciente. Así mismo por otro punto de vista o concepto, los proveedores de salud consideran la calidad de servicio el uso de los conocimientos y la tecnología usados en la atención. Los que administran los establecimientos de salud califican la calidad de servicio a la optimización de los recursos mientras se cumplen metas López et al (2019). Las diversas percepciones señaladas en el trabajo de López et al (2019) no se hallan alineadas a lo que la Declaración de Helsinki expresa en cuanto a la promoción, salvaguarda de la salud, el bienestar y los derechos de los pacientes como señala Cigarroa et al. (2018), en su investigación que la Asociación Médica Mundial desarrolló la Declaración de Helsinki como una declaración sobre los principios éticos para la investigación médica que involucra a participantes de la investigación humana y dirige a los médicos a promover y salvaguardar la salud, el bienestar y los derechos de los pacientes.

Es importante tener en cuenta al momento de querer evaluar las percepciones futuras en el manejo de los hospitales cuando se desea estudiar situaciones actuales o realidades de 
gestión para la toma de decisiones para el seguimiento, monitoreo o mejora; destacar la investigación de Pihlainen et al. (2019), quien sostiene y concluye, que existen escasos estudios donde se investigaron las percepciones del manejo futuro en los hospitales; y que mediante la adopción del método Argument Delphi pueden identificarse diferentes percepciones sobre la organización y orientación en el futuro del liderazgo y la gestión en los establecimientos hospitalarios; este aporte permite reflexionar en la medida que la revisión y corrección de instrumentos mediante juicio de expertos es esencial en la contribución para evaluar aspectos primordiales a medir para tener una validez de contenido metodológicamente coherente y tener resultados esperados del diagnóstico.

Por todo lo descrito y en consecuencia, se concluyó en la existencia de un problema: Deficiente nivel de desarrollo de los procesos de la gestión hospitalaria; formulándose el problema general de investigación siguiente ¿De qué manera la sistematicidad del plan integral de mejora fortalecerá la gestión hospitalaria a nivel institucional en la región San Martín, 2021?; también se planteó el objetivo general que orientó la investigación fue: Sistematizar el plan integral de mejora para fortalecer la gestión hospitalaria a nivel institucional en la región San Martín, 2021; y la hipótesis que responde al problema general dice: La sistematicidad del plan integral de mejora fortalecerá eficientemente la gestión hospitalaria a nivel institucional en la región San Martín, 2021.

Teóricamente los fundamentos que sostienen el estudio se rige en enfoques y marcos conceptuales, a continuación se detalla; que las instituciones hospitalarias por su funcionalidad operativa y de articulación estructural constituyen un sistema y a su vez una organización. Un sistema entiéndase bajo el término político al conjunto ordenado de normas y procedimientos que regulan el funcionamiento de un grupo o colectividad, o en términos gramaticales al conjunto de reglas, principios o medidas que tienen relación entre sí; en resumen es una integridad de un todo cuyas partes conforman piezas funcionales interrelacionados; Velásquez (2007), manifiesta que organización se refiere al establecimiento y agrupación de actividades y recursos necesarios que interactúan entre sí a través de una coordinación horizontal y vertical para el cumplimiento de los objetivos contemplados en la estructura de la empresa. Por tanto, agréguese al sistema el espacio o ambiente donde se desenvuelve la organización.

Para Morin, 1992 (Velásquez, 2007) "la organización es la disposición de relaciones entre componentes o individuos que produce una unidad compleja, dotado de cualidades 
desconocidas en el nivel de los componentes o individuos. La organización, pues, transforma, produce, reúne, mantiene". Dicho de otra forma, la organización es el conjunto de interrelaciones entre sus elementos constitutivos, es decir los roles o cargos, las cuales conforman una red cerrada que posee una identidad propia. Ocampo, 2007 (Velásquez, 2007) refiere que las organizaciones son entonces, formaciones sociales específicas en el tiempo y el espacio, desarrolladas por la sociedad como formas de articulación para resolver problemas concretos y que obran bajo propósitos específicos. La concepción de organización empresarial se redefine partiendo de cambios del nuevo siglo; como organizaciones que se basan en el conocimiento, en tecnología de la información y en mercados globales, para operar en grupos del conocimiento. La organización constituye un sistema orgánico implicado en un medio adverso con el que se realiza intercambio de energía, información y dinero, es decir, que la organización es un sistema socio-técnico abierto, el cual posee, in put (insumos), out put (productos) y retroalimentación o ciclos de retorno para modificar el propio sistema, en estructura, operación, función o propósito, lo que permite su persistencia en el tiempo, además de procesos internos de readaptación, construcción y autoreparación, permitiendo la interacción adecuada con su entorno.

Ante lo descrito y conceptualizado, los hospitales son organizaciones manejado por modalidades de gestiones diversas en su operatividad entre las que se encuentra la gestión hospitalaria; y que a continuación se detalla algunas teorías en las organizaciones; y que para efectos de comprender qué significa una Teoría, Sierra (1984) señala que, es toda concepción racional que intenta dar una visión o explicación sobre cualquier asunto o realidad; por su parte Carbajal (2002) también señala que teoría es el conjunto de enunciados que se encuentran conectados lógicamente entre sí, y forman una unidad epistemológica ordenada y sistemática que permite la explicación de la realidad, es decir, de las regularidades que suceden en el mundo real; y que contiene además leyes, hipótesis, definiciones y en casos más precisos axiomas, teoremas y ecuaciones entre otros. Por su parte, Washington (2013), expresa que una teoría por lo usual es expresada como una cadena de propuestas útiles en la explicación además de ser eventualmente predecible en aspectos de la realidad.

Sobre la Teoría de la gestión situacional. Se basa en la teoría de la contingencia de Mary Parker Follet 1920 donde plantea que cada organización es diferente y por lo tanto se 
requieren diferentes métodos de gerencia y administración; establece objetivos a corto, mediano y largo plazo (Meza, 2014). La teoría de las contingencias ignora de la existencia de una solución perfecta y presupone la búsqueda de solución de mejor conveniencia prestando atención a aspectos reales (Washington, 2013). Mientras, la teoría de la contingencia enfatiza que no hay nada absoluto en las organizaciones o en la teoría administrativa. Todo es relativo, todo depende (ULADECH, 2008). Contingencia, textualmente conceptualizado como algo eventual e incierto, que sucede o no sucede. La experiencia o la evidencia exponen la verdad o falsedad del conocimiento del fenómeno, mas no así por la razón. Por lo que como enfoque marca una nueva etapa en la Teoría General de la administración (Universidad Católica los Ángeles de Chimbote [ULADECH], 2008).

La gestión situacional, está basado en el enfoque de contingencias o método de enfoque situacional; es decir, aquel método donde se planifica tomar en consideración el tema situacional como también las circunstancias, a efectos que las decisiones sean determinadas adecuadamente; dicho y explicado de otra manera aquel cambio de la realidad por las situaciones del entorno, por tanto la teoría que tiene este enfoque toma en cuenta el medio ambiente en función de los cambios que se originan en el mismo y busca las modificaciones en el proceso de desarrollo organizacional. Por lo antes mencionado los que gerencian una institución como los gerentes, jefes y administradores tienen que definir qué técnicas, teorías o enfoque deben utilizar bajo determinada situación y circunstancias específicas para que las mismas permitan el cumplimiento de los objetivos que persiguen (Díaz, 2013). El enfoque contingencial manifiesta que las condiciones del ambiente están relacionados funcionalmente con las técnicas de la administración de manera apropiada para alcanzar eficazmente los objetivos organizacionales. (ULADECH, 2008).

Mientras que la Gestión por Procesos. Mallar (2010), señala que en la estructura organizativa se considera que toda organización es concebida como una red de procesos interrelacionados o interconectados, a la cual se puede aplicar un modelo de gestión denominado Gestión basado en Procesos. Ostroff, 2002 (Mallar, 2010), señala que el enfoque de la estructura organizativa vertical clásica, eficiente a nivel de funciones, se orienta hacia estructuras de tipo horizontal, además señala no existir contraposición de los modelos y donde cada organización empresarial busca equilibrio en referencia a sus 
innatas prioridades y posibilidades. Mallar (2010), señala que empresas líderes aplicaron el cambio organizativo, individualizando sus procesos, eligiendo los procesos relevantes, analizándolos y mejorándolos y finalmente utilizando este enfoque para transformar sus organizaciones.

Luego de los buenos resultados logrados, aplicaron la experiencia obtenida para optimizar el resto de sus procesos en toda la organización. El nuevo tipo de organización enfocada a los procesos, contiene sin embargo, a la anterior forma de organización estructural, sumándole el concepto del agregado de valor para un destinatario (cliente interno o externo) y exige atender, no solo a los factores internos del sistema (técnicos, etc.), sino también los requerimientos de dicha producción de valor. Esta finalidad es la misma que se considera en el método de Análisis del Valor como finalidad de satisfacción de necesidades del cliente. Con la visión panorámica de las teorías y conceptos de organización, sistemas y procesos; se pasa a destacar las denominaciones de plan y gestión en cuanto a lo que constituyen las variables del estudio en sí, haciendo referencia como organización a las instituciones hospitalarias.

En el marco conceptual del plan integral de mejora; en primer lugar se considera indispensable definir plan, como un conjunto de metas e instrumentos que tienen por finalidad la orientación de una determinada actividad humana en cierta dirección anticipada; mientras que integridad significa totalidad o global, hace referencia a un todo. Siguiendo el orden conceptual sumatorio, en segundo lugar, es importante conceptualizar al plan integral desde el enfoque del problema de la salud, y esta es definida introductoriamente por Amo y Rodríguez. (2010), como una serie de estrategias que intervienen de manera adecuada y ordenada para prevenir y manejar problemas, así como sus determinantes.

Además, el plan integral define estrategias que, en algunos casos, los servicios de salud los desarrollan en su ámbito propio, y en otros casos por instituciones o agentes sociales a través de la coordinación de acciones intersectoriales. Por lo que, abarca todas aquellas fases interventorías ante un problema diagnosticado de salud; a) promoción de activos protectores, b) prevención y control de factores de riesgo, c) diagnóstico precoz, d) calidad atención y e) incentivo de la recuperación del proyecto vital. Amo y Rodríguez (2010), sostienen, que un plan integral implica involucrar a todos los intervinientes, sanitarios y no sanitarios, que aporten su esfuerzo coordinadamente ante el problema. 
En el marco de la gestión hospitalaria, se precisa de los conocimientos conceptuales de la gestión de procesos, pues la gestión hospitalaria se basa en los mismos principios de fundamentos por ser las instituciones hospitalarias organizaciones que en su estructura de funcionamiento cumple procesos que deben ser ejecutados óptimamente para obtener resultados con calidad y satisfacción con todos los recursos disponibles para los que se planificaron. La gestión se conceptualiza como la interacción de todas las áreas de una empresa, organización, unidades, instituciones informativas, etc. Murray (2002) aduce que gestión es toda actividad dirigida a obtener y asignar los recursos necesarios para el cumplimiento de los objetivos de la organización.

Mientras que, Faga (2000), señala que, la gestión es una herramienta que engloba fases de la administración siendo la gestión misma un elemento con desarrollo propio de la administración. Mientras que Hospitalaria se conceptualiza, como aquel lugar que resulta agradable y acogedor para la persona que vive o está temporalmente en él; por lo que el significado de hospitalaria en el recinto sanitario viene a ser "aquel espacio que acoja al paciente y otorgue agradable confort durante su permanencia de atención de enfermedad". Bajo ese orden de términos conceptuales, la gestión hospitalaria se define como la función básica del proceso administrativo hospitalario que permite optimizar la oferta hospitalaria a una demanda de necesidades de atención de salud, en la que una gestión objetiva y una adecuada planeación posibilita la toma de decisiones y gestión administrativa de manera eficiente y oportuna (López y López, 2019). Ghasemi et al. (2017), menciona en su investigación que la revolución en la gestión hospitalaria requiere nuevos procedimientos $\mathrm{y}$ un cambio de paradigma en el mundo altamente competitivo. Los hospitales serán más exitosos al documentar cualquier sistema procedimiento bien organizado.

Sobre el Modelo, Caracheo, 2002 (Ramos et al. 2016) considera al modelo en términos generalizados como una representación de la realidad, explicación de un fenómeno, ideal digno de imitarse, paradigma, patrón, canon o guía de acción; idealización de la realidad; arquetipo, prototipo, uno entre una serie de objetos similares, un conjunto de elementos esenciales o los supuestos teóricos de un sistema social. También Aguilera, 2002 (Ramos et al. 2016) idealiza su concepción de modelo, como una representación parcial de la realidad; esto se refiere a que no es posible una explicación de la totalidad, ni incluir todas las variantes que esta pueda contener, por lo que se refiere más bien a la explicación de 
un fenómeno o proceso específico. Por tanto, de acuerdo a los conceptos de determinados autores; modelo, se define como una propuesta ideal, un patrón a imitar o seguir; pretendiendo así exponer las particularidades en general de la estructura del mencionado fenómeno; así como la explicación de sus componentes, medios y sucesiones, cómo son relacionados y los marcos teóricos que lo sustentan para ser fácil su comprensión. (Ramos et al., 2016).

Bajo este punto calificado como una pauta ideal a imitar o tomar; desde el punto de vista propio como autor del presente, califico como paradigma a todo modelo planteado en todos sus aplicativos prácticos. En ese sentido MINSA (2009) señala que el modelo de gestión hospitalaria, viene a ser el marco conceptual y operativo de referencia que define la forma de regular la gestión hospitalaria por el Estado, a través de un conjunto de políticas, sistemas, componentes procesos e instrumentos para que al operar coherentemente establecen la forma de conducir los servicios de salud del segundo y primer nivel de atención y dirigir su organización, permitiendo una adecuada y oportuna toma de decisiones para el logro de los objetivos sanitarios y económicos en el marco de una red de servicios de salud.

Mientras que para el MINSA (2011), los Lineamientos de Política de Salud 2007-2020 es base para el modelo de gestión, es ahí donde se plasma los principios obtenidos de la norma técnica documentaria del otrora modelo de atención integral de salud (MINSA, 2011) la integralidad, la universalidad, la calidad, la corresponsabilidad, la equidad, la solidaridad, la eficiencia. Así mismo en forma específica a los principios se consideraron siete lineamientos que dirigen al patrón de gestión hospitalaria que son:

- Lineamiento 1- Orientación al usuario,

- Lineamiento 2- La excelencia como base de la gestión hospitalaria en cuanto a su productividad social,

- Lineamiento 3- Responsabilidad de la autonomía contextualmente concertado e integrado en redes,

- Lineamiento 4- Gestión contractual,

- Lineamiento 5- Pluralismo financiero,

- Lineamiento 6- Participación social,

- Lineamiento 7- Transparencia y responsabilidad. 
En el logro de cambios de la gestión hospitalaria, el modelo actual MINSA (2009), señala que es necesario intervenir a nivel de tres actos de gestión con la finalidad de obtener el debido logro coherente e integral y la contundencia impactante en las operaciones de intervención. 1. Nivel Nacional y Regional, nivel que abarca la Macrogestión o Gestión Pública Hospitalaria, dichos niveles intervienen mediante el estado en la corrección de fallas del mercado y mejorando el bienestar social mediante la regulación en los aspectos de recursos humanos, tecnologías, financiamiento y organización en el marco de la gestión hospitalaria, 2. Nivel Hospitalario, nivel que implica directamente la Mesogestión o Gestión Institucional Hospitalaria, y que se refiere a la gestión institucional global hospitalaria; y 3. Nivel de Unidad Productora de Servicios de Salud, este nivel involucra la Microgestión o Gestión Clínica, y se refiere a la gestión de las Unidades Prestadoras de Servicio de Salud UPS al interior de cada hospital.

En el marco de cada nivel, el modelo se define en diez componentes; aquellos vienen a ser las líneas de intervención necesarias para modificar los procesos de gestión del hospital; y en cada proceso el modelo debe hacerse cumplir con el propósito de brindar una mejor calidad de atención con satisfacción del usuario con el uso racional de los recursos. Para efectos de la investigación ubicándonos en la realidad institucional carente de información local a nivel de los procesos, además gestores de su propio desenvolvimiento que resulta en un desarrollo adecuado o inadecuado; los componentes serán estudiados a nivel hospitalario o institucional, se describen cada uno según su marco conceptual, son diez los identificados: 1. Gestión clínica, 2. Gestión administrativa, 3. Gestión de recursos humanos, 4. Gestión de recursos tecnológicos, 5. Gestión del sistema de información, 6. Investigación y docencia, 7. Financiamiento y mecanismo de pago, 8. Plataforma organizativa, 9. Plataforma estratégica, 10. Plataforma jurídica.

\section{MATERIALES Y METODOS}

El tipo de investigación aplicado fue de tipo básica con el enfoque cuantitativo. A su vez, la investigación también fue de tipo proyectiva porque propone una solución del problema. Al respecto, Hurtado (2008) señala que un estudio es proyectivo, cuando propone soluciones a una determinada situación partiendo de una indagación, exploración, descripción, explicación y propone alternativas de cambio, pero, no necesariamente se ejecutan. El tipo diseño fue no experimental a nivel descriptivo; describió la realidad de la situación actual de la gestión hospitalaria en la región San 
Martín, se analizó y finalmente se diseñó la propuesta que responde a la realidad y situación actual encontrada. Según Chávez (2007) el diseño no experimental permite observar los fenómenos y cómo estos se desarrollan en su estado natural; sin la manipulación de las variables. Para la obtención muestral se empleó la fórmula para población finita $n=\frac{N Z^{2} p \cdot q}{E^{2}(N-1)+Z^{2} p \cdot q}$, obteniendo 110 colaboradores profesionales $\mathrm{y}$ técnicos entre administrativos y asistenciales. El muestreo fue de tipo probabilístico estratificado; conociendo la proporción elegida para aplicar el instrumento de acuerdo a cuatro estratos identificados: Estrato 1: Profesionales asistenciales. 289. Estrato 2: Profesionales administrativos 16. Estrato 3: Técnicos asistenciales 178. Estrato 4: Técnicos administrativos 59. Aplicando la siguiente fórmula: $n e=n i / N * n$, obteniendo la distribución muestral en cada estrato fue el siguiente: 59 fueron asistenciales profesionales, 3 fueron administrativos profesionales, 36 fueron asistenciales técnicos, y 12 fueron administrativos técnicos. No obstante, el investigador seleccionó la muestra de acuerdo a los siguientes criterios para garantizar la fiabilidad de las respuestas: - Por la confiabilidad de la información. - Por la accesibilidad a la información. - Porque forman parte del personal en la toma de decisiones. - Conocen los procesos administrativos y asistenciales del Hospital Es Salud Tarapoto; además se consideró factible ser responsable de área y/o departamento. Técnica de recolección fue la encuesta y el instrumento aplicado fue un cuestionario estructurado con 48 ítems, que correspondieron a una interrogante de cada indicador perteneciente a las diez dimensiones de la gestión hospitalaria; dicho instrumento fue elaborado por el propio investigador y los ítems fueron desarrollados de acuerdo a los puntos de evaluación de los procesos de los diez componentes de la gestión hospitalaria del modelo de gestión hospitalaria del MINSA (2009); se mantuvieron correlación metodológica en cada uno de ellos con tal de mantener la correlación entre los indicadores, dimensiones y la variable del estudio. Para la validación del instrumento se aplicó la técnica de juicio de expertos y no pilotaje porque las medidas del distanciamiento social aún se mantuvieron en la entidad hospitalaria donde se ejecutó dicho instrumento. Para la confiabilidad se aplicó el calculó mediante la técnica del Alfa de Cronbach resultando en 0.7, ubicándose en el rango de 0.61 a 0.8 de magnitud alta; concluyendo, que el instrumento aplicado para diagnosticar el estado situacional de la gestión hospitalaria a nivel institucional es de confiabilidad alta. La investigación se desarrolló en tres etapas como procedimiento; primera etapa, en esta 
etapa se realizó la fase diagnóstica mediante un diseño descriptivo simple; porque no se pretendió comparar, correlacionar o explicar una causa; sólo se necesita fundamentar la comprensión del fenómeno. Para efectos de la recolección de la información, se diseñó un instrumento con un cuestionario de preguntas la que fue validada por cinco expertos y se ejecutó vía virtual con colaboradores de una institución hospitalaria por medidas sanitarias del COVID. Los resultados de esta fase fueron que la gestión hospitalaria a nivel institucional fue de nivel regular, más no eficiente; permitiéndonos analizar que la hipótesis general no es eficiente; segunda etapa; en base al diagnóstico observado de nivel regular y no el óptimo o eficiente de la gestión hospitalaria a nivel institucional y los antecedentes observados en otros estudios, añadido las teorías relacionadas al sistema, la administración y las contingencias que aclaran que toda organización se desarrolla bajo sistemas y que sus componentes se interrelacionan y son interdependientes; y contingencialmente existen diferentes métodos de gerencia y administración; conllevó a planificar el diseño de un plan integral de mejora; plan, porque se desarrollará un modo o conjunto de acciones necesarios para lograr la idea; integral, porque las bases de la administración de Fayol sostiene que el enfoque sistémico integral abarca todas las esferas de las empresas y que la organización y sus componentes son interdependientes; mejora, porque los sistemas predicen patrones de comportamiento, y el cambio de una parte del sistema afecta a los demás; en efecto bajo la propuesta del plan integral de mejora se pretende fortalecer la gestión hospitalaria a nivel institucional en la región San Martín, 2021. Esta fase es la denominada fase proyectiva porque solo constituye una propuesta de solución, mas no se ejecutará o aplicará, como afirma (Hurtado, 2008); tercera etapa, la propuesta diseñada se llevó a una opinión de consulta de cinco expertos con grado de Doctor quienes evaluaron la propuesta del plan integral de mejora para fortalecer la gestión hospitalaria a nivel institucional en la región San Martín, 2021, en base a un instrumento elaborado y estructurado en tres componentes como son la sistematicidad, la contribución y la prospectiva las cuales reportaron calificaciones valorativas cuantitativas; y además una calificación de tipo cualitativa en el subcomponente de sistematicidad; es decir, la apreciación del plan mencionando donde requiere mejorar o modificar, y detallando la sugerencia de mejora. En cuanto a las sugerencias hechas por los expertos, se mejoró el diseño de la propuesta consolidando así su prospectiva. La gestión del cambio en la propuesta surte como entrada el diagnostico, 
las teorías y los marcos legales, la fase de proceso son las acciones de medidas a considerar en el plan donde intervienen todos los componentes administrativos y asistenciales con responsables respectivos y un sistema programado de seguimiento y evaluación; y por último en su fase de resultados se espera lograr los diez componentes con gran nivel de desarrollo apoyado con el seguimiento y monitoreo según cronograma fortalecer la gestión hospitalaria a nivel de la mesogestión en San Martín con el objetivo de optimizar el nivel de desarrollo para lograr mejores indicadores de gestión que resultan en el buen servicio y calidad de atención. Para el análisis de datos se empleó la estadística descriptiva, en ese sentido se consideró las unidades de tendencia central la media, y las medidas de dispersión la desviación estándar. Los datos fueron procesados en el programa estadístico SPSS versión 26 y la información se consignó en el formato Word y Excel para su presentación mediante tablas de frecuencia.

\section{RESULTADOS Y DISCUSIÓN}

Los componentes de la gestión hospitalaria reportaron las siguientes escalas de desarrollo: gestión clínica una escala de desarrollo de nivel regular con el $72 \%$ correspondiendo a 79 encuestados, gestión administrativa una escala de desarrollo de nivel regular con el 63\% representados por 69 encuestados, gestión de recursos humanos con una escala de desarrollo de nivel regular con el 85\% representados en 93 encuestados, gestión de recursos tecnológicos con una escala de desarrollo de nivel regular con el $66 \%$ representados en 73 encuestados, gestión de los sistemas de información con una escala de desarrollo de nivel regular con el 69\% representados en 76 encuestados, investigación y docencia con una escala de desarrollo de nivel regular con el 57\% representados en 63 encuestados, financiamiento y/o mecanismo de pago con una escala de desarrollo de nivel regular con el $81 \%$ representados en 89 encuestados, plataforma organizativa con una escala de desarrollo de nivel regular con el 68\% representados en 75 encuestados, plataforma estratégica con una escala de desarrollo de nivel regular con el 58\% representados en 64 encuestados y por último la plataforma jurídica con una escala de desarrollo de nivel regular con el 66\% representados en 73 encuestados; observando en efecto que la situación actual de la gestión hospitalaria en la región San Martín, 2021 se encuentra en un nivel de desarrollo de cumplimiento de los procesos institucionales en una escala valorativa de "Regular" con un 78\%, seguido de una gestión de nivel eficiente (18\%) y nivel deficiente (4\%); por lo que, se observa que no es eficiente. 
Tabla 1 Gestión hospitalaria a nivel institucional en San Martín.

\begin{tabular}{lccc}
\hline Escala & Rango & Cantidad & Porcentaje \\
\hline Deficiente & $48-111$ & 4 & $4 \%$ \\
Regular & $112-175$ & 86 & $78 \%$ \\
Eficiente & $176-240$ & 20 & $18 \%$ \\
Total & & $\mathbf{1 1 0}$ & $\mathbf{1 0 0 \%}$ \\
\hline
\end{tabular}

Fuente: Base de datos del cuestionario aplicado a 110 colaboradores.

Al ser la propuesta evaluado por los siete expertos sobre la sistematicidad, coherencia y consistencia entre los componentes que estructuran el Plan integral de mejora; se evidenció que, el 46\% de las opiniones (32) consideran como muy adecuado; un $40 \%$ de las versiones (28) calificaron como bastante adecuado, un 13\% de las opiniones (9) calificaron como adecuado y sólo $1 \%$ de los que opinaron (1) calificó como poco adecuado. En efecto, el mencionado plan expresa la evidencia de ser consistente y coherente dentro su estructura.

Tabla 2 Sistematicidad y consistencia del Plan integral de mejora para fortalecer la gestión hospitalaria a nivel institucional en la región San Martín, 2021.

\begin{tabular}{|c|c|c|c|c|c|c|c|c|c|c|c|c|}
\hline \multicolumn{2}{|c|}{ Principales componentes } & 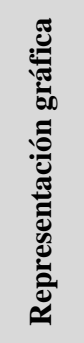 & 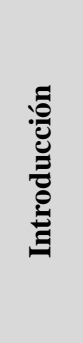 & 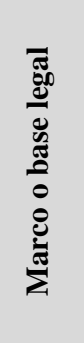 & 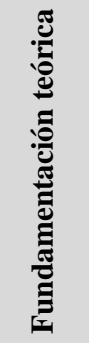 & 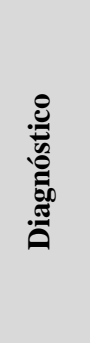 & 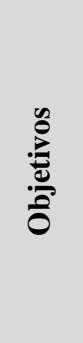 & 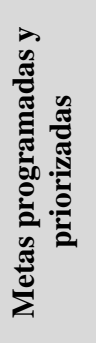 & 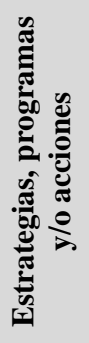 & 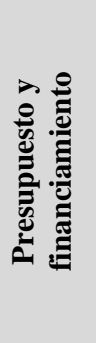 & 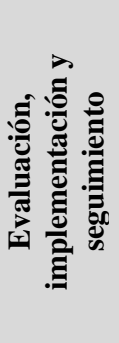 & 是 \\
\hline \multirow{2}{*}{ Muy adecuado } & Cantidad & 2 & 4 & 4 & 4 & 5 & 4 & 2 & 3 & 2 & 2 & 32 \\
\hline & Porcentaje & $29 \%$ & $57 \%$ & $57 \%$ & $57 \%$ & $71 \%$ & $57 \%$ & $29 \%$ & $43 \%$ & $29 \%$ & $29 \%$ & $46 \%$ \\
\hline \multirow{2}{*}{$\begin{array}{l}\text { Bastante } \\
\text { adecuado }\end{array}$} & Cantidad & 3 & 2 & 3 & 2 & 2 & 2 & 4 & 4 & 3 & 3 & 28 \\
\hline & Porcentaje & $43 \%$ & $29 \%$ & $43 \%$ & $29 \%$ & $29 \%$ & $29 \%$ & $57 \%$ & $57 \%$ & $43 \%$ & $43 \%$ & $40 \%$ \\
\hline \multirow{2}{*}{ Adecuado } & Cantidad & 1 & 1 & 0 & 1 & 0 & 1 & 1 & 0 & 2 & 2 & 9 \\
\hline & Porcentaje & $14 \%$ & $14 \%$ & $0 \%$ & $14 \%$ & $0 \%$ & $14 \%$ & $14 \%$ & $0 \%$ & $29 \%$ & $29 \%$ & $13 \%$ \\
\hline \multirow{2}{*}{ Poco adecuado } & Cantidad & 1 & 0 & 0 & 0 & 0 & 0 & 0 & 0 & 0 & 0 & 1 \\
\hline & Porcentaje & $14 \%$ & $0 \%$ & $0 \%$ & $0 \%$ & $0 \%$ & $0 \%$ & $0 \%$ & $0 \%$ & $0 \%$ & $0 \%$ & $1 \%$ \\
\hline \multirow{2}{*}{ Inadecuado } & Cantidad & 0 & 0 & 0 & 0 & 0 & 0 & 0 & 0 & 0 & 0 & 0 \\
\hline & Porcentaje & $0 \%$ & $0 \%$ & $0 \%$ & $0 \%$ & $0 \%$ & $0 \%$ & $0 \%$ & $0 \%$ & $0 \%$ & $0 \%$ & $0 \%$ \\
\hline \multirow{2}{*}{ TOTAL } & Cantidad & 7 & 7 & 7 & 7 & 7 & 7 & 7 & 7 & 7 & 7 & 70 \\
\hline & Porcentaje & $100 \%$ & $100 \%$ & $100 \%$ & $100 \%$ & $100 \%$ & $100 \%$ & $100 \%$ & $100 \%$ & $100 \%$ & $100 \%$ & $100 \%$ \\
\hline
\end{tabular}

Fuente: Elaboración propia a partir del informe de opinión de los expertos. 
En lo que se refiere al grado de contribución que presenta el plan integral de mejora en el fortalecimiento de la gestión hospitalaria a nivel institucional en la región San Martín; en la evaluación de los expertos resultó que el 70\% de las valoraciones (49) sostienen que el plan se atribuye un excelente grado de contribución para resolver el problema de la gestión hospitalaria; Así mismo, un 30\% de las valoraciones (21); consideran que el plan tiene un buen grado de contribución; para fortalecer la gestión hospitalaria.

Tabla 3 Contribución del Plan integral de mejora del Plan integral de mejora para fortalecer la gestión hospitalaria a nivel institucional en la región San Martín, 2021.

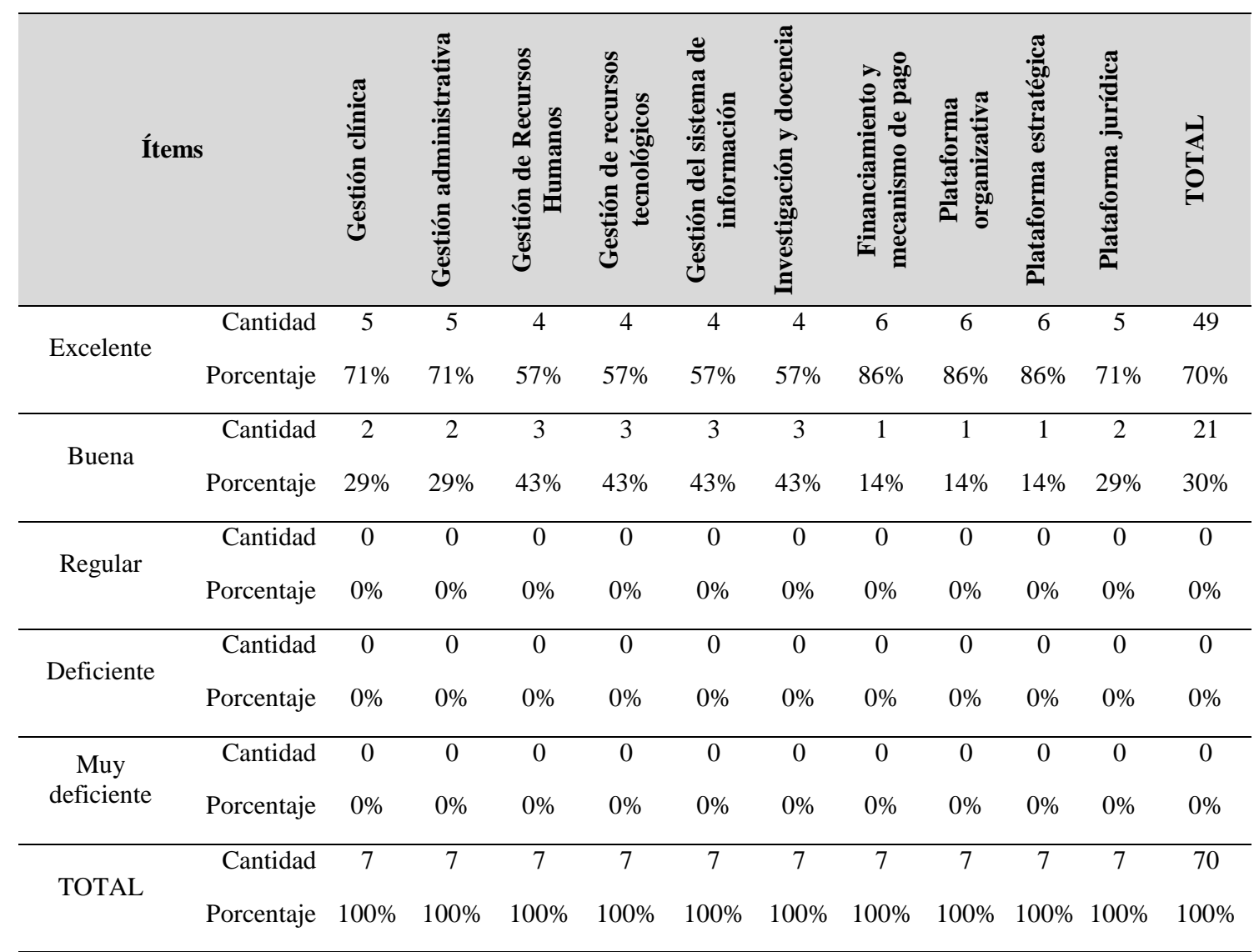

Fuente: Elaboración propia a partir del informe de opinión de los expertos.

Por último, el consolidado de las opiniones de los siete expertos con respecto a la prospectiva de poder implementarse el plan integral de mejora; se observó que el $60 \%$ en las valoraciones (21) coincidieron en calificar como de excelente la posibilidad de implementarse el mencionado plan; así mismo, el 40\% de las valoraciones (14) señalan que la prospectiva del plan es buena. En efecto, existe la deducción que la referida 
propuesta posee una excelente prospectiva; por cuanto, es considerado ser viable, factible, aplicable, sostenible y pertinente.

Tabla 4 Prospectiva del Plan integral de mejora del Plan integral de mejora para fortalecer la gestión hospitalaria a nivel institucional en la región San Martín, 2021.

\begin{tabular}{|c|c|c|c|c|c|c|c|}
\hline Criterios & & 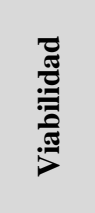 & 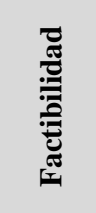 & 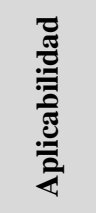 & 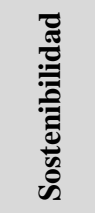 & 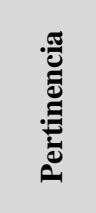 & 总 \\
\hline \multirow{2}{*}{ Excelente } & Cantidad & 5 & 4 & 4 & 3 & 5 & 21 \\
\hline & Porcentaje & $71 \%$ & $57 \%$ & $57 \%$ & $43 \%$ & $71 \%$ & $60 \%$ \\
\hline \multirow{2}{*}{ Buena } & Cantidad & 2 & 3 & 3 & 4 & 2 & 14 \\
\hline & Porcentaje & $29 \%$ & $43 \%$ & $43 \%$ & $57 \%$ & $29 \%$ & $40 \%$ \\
\hline \multirow{2}{*}{ Regular } & Cantidad & 0 & 0 & 0 & 0 & 0 & $\mathbf{0}$ \\
\hline & Porcentaje & $0 \%$ & $0 \%$ & $0 \%$ & $0 \%$ & $0 \%$ & $0 \%$ \\
\hline \multirow{2}{*}{ Deficiente } & Cantidad & 0 & 0 & 0 & 0 & 0 & $\mathbf{0}$ \\
\hline & Porcentaje & $0 \%$ & $0 \%$ & $0 \%$ & $0 \%$ & $0 \%$ & $0 \%$ \\
\hline \multirow{2}{*}{ Muy deficiente } & Cantidad & 0 & 0 & 0 & 0 & 0 & $\mathbf{0}$ \\
\hline & Porcentaje & $0 \%$ & $0 \%$ & $0 \%$ & $0 \%$ & $0 \%$ & $0 \%$ \\
\hline \multirow{2}{*}{ TOTAL } & Cantidad & 7 & 7 & 7 & 7 & 7 & 35 \\
\hline & Porcentaje & $100 \%$ & $100 \%$ & $100 \%$ & $100 \%$ & $100 \%$ & $100 \%$ \\
\hline
\end{tabular}

Fuente: Elaboración propia a partir del informe de opinión de los expertos.

La calificación de la sistematicidad, contribución y prospectiva del plan integral de mejora obtenidos fueron: adecuado y bastante adecuado con el $86 \%$ con respecto a la sistematicidad y coherencia; de excelente y bueno con el 100\% el grado de contribución y la prospectiva (Ver tablas 2, 3 y 4). Al respecto, Ghasemi et al. (2017) manifiesta que la gestión hospitalaria requiere nuevos procedimientos y un cambio de paradigma en el mundo altamente competitivo. Además, los hospitales serán más exitoso al documentar cualquier sistema de procedimiento bien organizado (Ghasemi et al. 20217). Ante este aporte del mencionado autor, el plan integral de mejora constituye un nuevo procedimiento como parte de una reforma para mejorar el desenvolvimiento de la gestión hospitalaria. Por tanto, las altas valoraciones contribuyen en la mejora y fortalecimiento de los procesos de la gestión hospitalaria institucional.

Sin embargo, merece discutir como crítica a la manera de haberse ejecutado la recolección de la información, como la existencia de barreras o limitaciones establecidos por el establecimiento hospitalario en cuanto a la solicitud de participación de los colaboradores porque existía de por medio una disposición de no permitir atenciones con prestación 
física por la coyuntura del COVID, sugiriendo realizar vía virtual; esta limitación originó el reto de evaluar la manera cómo entablar comunicación con el colaborador a fin de dar a conocer sobre el contenido y propósito de la encuesta a resolver; pues sólo existió la vía telefónica; sin embargo, los resultados obtenidos probablemente no fueron los esperados debido a que el interés de participar y su adherencia de compromiso no fueron los ideales. También cabe señalar que los ítems respondidos entre la valoración regular y adecuado de la encuesta que resultó en una situación actual de regular en las diez dimensiones, hayan intervenido percepciones para responder a favor de la situación del hospital como gestión y no revelar la situación real que probablemente haya sido la más ineficiente.

\section{CONCLUSIONES}

El grado de sistematicidad, contribución y prospectiva del plan integral de mejora para fortalecer la gestión hospitalaria a nivel institucional en la región San Martín, 2021, es adecuado y excelente según la opinión de los expertos; es decir, la prospectiva de poder aplicarse en la mejora de la gestión hospitalaria sería sorprendentemente viable, factible, aplicable, sostenible y pertinente.

\section{LISTADE REFERENCIAS}

Alvitres, V. (2000). Método Científico, Planificación de la Investigación (Editorial Ciencias (ed.)).

Arias, F. G. (2012). El Proyecto de Investigación Introducción a la metodología científica (E. Episteme (ed.); Sexta edic).

Amo Alfonso, M., \& Rodríguez Rodríguez, M. (2010). Metodología de elaboración y seguimiento de planes integrales de salud. https://enfermeriacomunitaria.org/web/attachments/article/516/Planes Integrales de Salud.pdf

Arasteh, M. A., Shamshirband, S., \& Yee, P. L. (2018). Using multi-attribute decisionmaking approaches in the selection of a hospital management system. Technology and Health Care, 26(2), 279-295. https://doi.org/10.3233/THC-170947

Bastani, P. et al. (2021). Hospital management by health services management graduates: the change paradigm in Iran. Heliyon 7 (2021) e08414. https://doi.org/10.1016/j.heliyon.2021.e08414

Bernal, C. A. (2010). Metodología de la investigación (Tercera (ed.); 3rd ed.). PEARSON EDUCACIÓN. 
Carvajal Villaplana, Á. (2002). Teorías y modelos: formas de representaciones de la Una teoría es usualmente expresada como una serie de proposiciones que sirven para explicar y eventualmente predecir aspectos de la realidad. Comunicación, 12(1), 1-14. https://www.redalyc.org/pdf/166/16612103.pdf

Cigarroa, F. G., Masters, B. S., \& Sharphorn, D. (2018). Institutional Conflicts of Interest and Public Trust. JAMA, 320(22), 2305. https://doi.org/10.1001/jama.2018.18482

Concytec. (2018). Reglamento de calificación, clasificación y registro de los investigadores del sistema nacional de ciencia, tecnología e innovación tecnológica - reglamento RENACYT. portal.concytec.gob.pe. https://portal.concytec.gob.pe/images/renacyt/reglamento_renacyt_version_final. pdf

Díaz, A. (2013). Teorías de la administración. Sites.Google.Com. https://sites.google.com/site/gestionalejandrodc96/teorias-de-la-administracion

Díaz Álvarez, J. C. (2021). Modelos de gestión hospitalaria y su influencia en la calidad de atención al usuario del servicio de salud: revisión sistemática rápida de la literatura [Universidad EAN]. https://repository.ean.edu.co/handle/10882/10484

Faga, R. (2000). Cómo profundizar en el análisis de sus costos para tomar mejores decisiones empresariales (Granica (ed.)).

Flores, J. (2021). Gestión hospitalaria: Una mirada al desarrollo de sus procesos. Ciencia Latina Revista Científica Multidisciplinaria.

\section{https://ciencialatina.org/index.php/cienciala/article/view/368/458}

Ghasemi, M., Ghadiri Nejad, M., \& Bagzibagli, K. (2017). Knowledge Management Orientation: An Innovative Perspective to Hospital Management. Iranian journal of public health, 46(12), 1639-1645. http://www.ncbi.nlm.nih.gov/pubmed/29259938

Gordillo Tobar, A., \& Herrera Rodríguez, E. (2020). Desarrollo de los Recursos Humanos para el fortalecimiento institucional del Ministerio de Salud de Nicaragua. Knowledge Brief. http://hdl.handle.net/10986/35088

Gu, D., Deng, S., Zheng, Q., Liang, C., \& Wu, J. (2019). Impacts of case-based health knowledge system in hospital management: The mediating role of group effectiveness. Information \& Management, 56(8), 103162. https://doi.org/10.1016/j.im.2019.04.005 
Henríquez Espejo, M. (2020). Eficiencia del gasto hospitalario en Chile. https://observatoriofiscal.cl/archivos/documento/20200816122601070.pdf

Hernandez Sampieri, R., Fernandez Collado, C., \& Baptista Lucio, P. (2014). Metodología de la Investigación (Mc Graw Hill Education. (ed.); 6th ed.).

Hurtado de Barrera, J. (03 de abril de 2008). Algunos criterios metodológicos de la investigación. http://investigacionholistica.blogspot.com/2008/04/algunoscriterios-metodolgicos-de-la.html

Livinus, V., Adhikara, M. F. A., \& Kusumapradja, R. (2021). Hospital Management Information System Usefulness in The Health Services Industry at Indonesia: Mandatory or Voluntary? JMMR (Jurnal Medicoeticolegal dan Manajemen Rumah Sakit), 10(1), LAYOUTING. https://doi.org/10.18196/jmmr.v10i1.10296

López Jara, A. A., \& López Vintimilla, X. R. (2019). Indicadores para evaluar la eficiencia hospitalaria. Caso de estudio: clínica privada de la ciudad de Macas; Uniandes EPISTEME. Revista Digital de Ciencia, Tecnología e Innovación, 6(3), 383-398. http://45.238.216.13/ojs/index.php/EPISTEME/article/view/1401

López, P., \& Fachelli, S. (2015). Metodología de la investigación social cuantitativa. Universidad Autónoma de Barcelona.

Mallar, M.A. (2010). La Gestión por Procesos: Un enfoque de gestión eficiente. Argentina. Revista Científica "Visión de Futuro"

Méndez, C., \& Torres, C. (2010). Autonomía en la gestión hospitalaria en Chile: los desafíos para el recurso humano en salud. Instituto de Salud Pública. https://scielosp.org/pdf/rsp/2010.v44n2/366-371/es

Mei, J., \& Kirkpatrick, I. (2019). Public hospital reforms in China: towards a model of new public management? International Journal of Public Sector Management, 32(4), 352-366. https://doi.org/10.1108/IJPSM-03-2018-0063

Meza, L. (2014). Teorías de gestión. Mindomo.Com. https://www.mindomo.com/es/mindmap/teorias-de-gestion022525ff16454b518b73d31c74561011

Ministerio de Salud. (2009). Modelo de gestión hospitalaria. Dirección general de salud de las personas. https://www.saludarequipa.gob.pe/goyeneche/doc/NORMAS/NORMATIVA_E N_SALUD/25B Lineamientos de Gestion Hospitalaria 
29102009_anteproyecto_2009.pdf

Ministerio de Salud. (2011). Modelo de atención integral de salud basado en familia y comunidad. Dirección general de salud de las personas. https://determinantes.dge.gob.pe/archivos/1880.pdf

Murray, P. (2002). Gestión - Información - Conocimiento. Biblios, 14. https://www.redalyc.org/pdf/161/16114402.pdf

Naranjo-Gil, D. (2016). Cómo los equipos de dirección usan los sistemas de información y control en la gestión hospitalaria. Gaceta Sanitaria, 30(4), 287-292. https://doi.org/10.1016/j.gaceta.2015.12.009

Navarro Luna, S. L., \& Gambetta Rios, P. F. (2018). Gestión Hospitalaria y Calidad de los Registros de las Historias Clínicas del Servicio de Odontología en ESSALUD de la Región Tacna, año 2018 [Universidad César Vallejo]. https://repositorio.ucv.edu.pe/handle/20.500.12692/34742

Palella, S., \& Martins, F. (2012). Metodología de la Investigación Cuantitativa (Tercera). Pihlainen, V., Kivinen, T., \& Lammintakanen, J. (2019). Perceptions of future hospital management in Finland. Journal of Health Organization and Management, 33(5), 530-546. https://doi.org/10.1108/JHOM-02-2018-0045

QuestionPro. (2020).¿Qué es un cuestionario? Questionpro.Com. https://www.questionpro.com/blog/es/que-es-un-cuestionario/

Ramos Ríos, M.S., Vargas Atencio, Y, Barrios Martínez, A. \& Cifuentes Cifuentes, O. A. (2016). Modelo conceptual de turismo sostenible para el corregimiento de San Basilio de Palenque departamento de Bolivar Colombia. https://dialnet.unirioja.es/servlet/articulo?codigo $=6577486$

Reynaga Salazar, S.L. (2019). Gestión hospitalaria en la calidad de servicio del Hospital Nacional Cayetano Heredia, 2019 [Universidad César Vallejo].

Rios Lavi, G. (2016). Modelo de gestión hospitalaria y valores éticos y morales en el RR. HH. del Hospital II -1 Rioja 2014 [Universidad César Vallejo]. https://repositorio.ucv.edu.pe/bitstream/handle/20.500.12692/26006/Rios_LG.pd $\mathrm{f}$ ? sequence $=1 \&$ isAllowed $=\mathrm{y}$

Saeed Okour, K. (2020). The Impact of Total Quality Management on the Quality of Institutional Performance in Saudi Hospitals: A Case Study of King Faisal Hospital. International Journal of Psychosocial Rehabilitation, 24(3). 
https://www.researchgate.net/profile/Karim-

Okour/publication/339432433_The_Impact_of_Total_Quality_Management_on _the_Quality_of_Institutional_Performance_in_Saudi_Hospitals_A_Case_Study _of_King_Faisal_Hospital/links/5f3e878e458515b72931f401/The-Impact-ofTotal-Quality-Management-on-the-Quality-of-Institutional-Performance-inSaudi-Hospitals-A-Case-Study-of-King-Faisal-Hospital.pdf

Saturno Hernández, P., Hernández Avila, M., Magaña Valladares, L., García Saisó, S., \& Vertiz Ramirez, J. de J. (2015). Estrategia integral de formación para la mejora continua de la calidad de los servicios de salud. Salud pública Méx, 57(3). http://www.scielo.org.mx/scielo.php?script=sci_arttext\&pid=S003636342015000300017

Sierra Bravo, R. (1984). Ciencias sociales, epistemología, lógica y metodología (Paraninfo (ed.); 1st ed.).

Suárez, J. (2012). Institutional plan for solid waste management, a tool for hospital management. Rev Cubana Hig Epidemiol, 50(3). https://www.medigraphic.com/cgibin/new/resumenI.cgi?IDREVISTA=253\&IDARTICULO=43726\&IDPUBLIC $\mathrm{ACION}=4544$

Terán, G.J. et al. (2017). Gestión de la innovación en los servicios de salud pública. Revista Cubana de Investigaciones Biomédicas. 2017; 36(3). http://scielo.sld.cu/pdf/ibi/v36n3/ibi16317.pdf

Universidad Católica los Ángeles de Chimbote. (2008). Teoría contingencial. Files.Uladech.Edu.Pe/.

http://files.uladech.edu.pe/docente/32805306/ADMINISTRACION_I/Sesion_12 /Contenido_Sesion12.pdf

Velásquez, A. (2007). La organización, el sistema y su dinámica: Una versión desde Niklas Luhmann. Revista Escuela de Administración de Negocios, núm. 61, septiembre-diciembre, 2007, 129-155. https://www.redalyc.org/pdf/206/20611495014.pdf

Washington Barrientos, J. (2013). Teoría de las contingencias múltiples dinámicamente entrelazadas abriendo la caja negra del ajuste contingente. Ciencias Administrativas, 2, 21-32. https://www.redalyc.org/pdf/5116/511651378004.pdf 